\title{
Strain hardening and ductility in a coarse-grain/nanostructure laminate material
}

\author{
X.L. Ma, ${ }^{a}$ C.X. Huang, ${ }^{b, *}$ W.Z. Xu, ${ }^{a}$ H. Zhou, ${ }^{a, c}$ X.L. Wu ${ }^{d}$ and Y.T. Zhu ${ }^{a, e, *}$ \\ ${ }^{a}$ Department of Materials Science and Engineering, North Carolina State University, Raleigh, NC 27695, USA \\ ${ }^{\mathrm{b}}$ School of Aeronautics and Astronautics, Sichuan University, Chengdu 610065, China \\ ${ }^{\mathrm{c}}$ National Engineering Research Center of Light Alloy Net Forming, Shanghai Jiao Tong University, Shanghai 200240, China \\ ${ }^{\mathrm{d}}$ State Key Laboratory of Nonlinear Mechanics, Institute of Mechanics, Chinese Academy of Sciences, Beijing 100190, China \\ ${ }^{\mathrm{e}}$ School of Materials Science and Engineering, Nanjing University of Science and Technology, Nanjing 210094, China
}

Received 26 December 2014; revised 10 February 2015; accepted 15 March 2015

Available online 26 March 2015

\begin{abstract}
A laminate structure with a nanostructured $\mathrm{Cu}-10 \mathrm{Zn}$ layer sandwiched between two coarse-grained $\mathrm{Cu}$ layers was produced by high-pressure torsion, rolling and annealing. Sharp interlayer interfaces with sufficient bonding strength were developed. Mechanical incompatibility between different layers during tensile deformation produced high strain hardening, which led to a tensile ductility higher than prediction by the rule-of-mixture. These observations provide insights into the architectural design and deformation studies of materials with gradient and laminate structures. Published by Elsevier Ltd. on behalf of Acta Materialia Inc.
\end{abstract}

Keywords: Laminate structure; Strain hardening; Ductility; Nanostructure; Interface

Gradient structures (GS) with a grain-size gradient have been recently introduced to optimize the mechanical properties of structural materials [1-5]. To date, some exceptional combination of enhanced strength and considerable ductility are reported in different material systems with multiscale grain-size structures [6-10], including GS and multi-layered materials. However, the fundamental principles that govern the deformation behaviors of GS are still not fully understood [1,5]. Elastic/plastic interface and stable/unstable interface caused by mechanical incompatibility during deformation have been reported to play a critical role in both strengthening and strain hardening of GS materials [2,5]. However, such interfaces migrate dynamically during the deformation of GS, which makes it hard to perform quantitative postmortem investigation $[1,2,11]$. In fact, GS can be approximately regarded as the integration of numerous layers and interfaces (laminate structure) [2]. Therefore, there are some similarities in the deformation behaviors of GS and laminate structures. For example, both structures have the mechanical incompatibility during deformation. The advantage of laminate structure is that its interfaces are stationary [12] and can be easily located after the deformation, making it easier

\footnotetext{
* Corresponding authors at: School of Aeronautics and Astronautics, Sichuan University, Chengdu 610065, China (C.X. Huang); Department of Materials Science and Engineering, North Carolina State University, Raleigh, NC 27695, USA (Y.T. Zhu).; e-mail addresses: chxhuang@scu.edu.cn; ytzhu@ncsu.edu
}

to analyze quantitative mechanics and investigate postmortem microstructures. Therefore, it might be possible to use laminated (or sandwiched) structures to study some fundamentals in deformation behaviors of GS structure.

The fabrication of laminated nanostructured (NS)/coarse-grained (CG) structures with sharp interfaces is also a challenge since interfacial strength and selective grain refinement are required simultaneously. Here, we fabricated a laminate structure with a NS bronze layer sandwiched between two CG copper layers by utilizing two principles: (a) Different grain refinement effectiveness of $\mathrm{Cu}$ and bronze during deformation. It has been shown that the grain size of bronze can be refined much more effectively than that of copper during severe plastic deformation due to its lower stacking-fault energy [13]. (b) Different thermal stabilities of $\mathrm{Cu}$ and bronze. Alloying elements are generally effective in pinning grain boundaries and resisting grain growth [14]. Hence, bronze can remain much finer grain size compared to $\mathrm{Cu}$ after proper annealing. Using this approach, we can produce a laminate structure with sharp and well-bonded NS/CG interfaces. Another objective of this work is to use NS/CG sandwich to study the effect of grain-size-difference across the interfaces on mechanical behaviors.

Figure 1a schematically illustrates the procedure of sample processing. Commercial $\mathrm{Cu}(99.9$ wt.\%) and bronze $(\mathrm{Cu}-10 \mathrm{wt} . \% \mathrm{Zn})$ plates were punched into $\phi-10 \mathrm{~mm}$ disks and polished to 3 groups of thickness. The total initial thickness of three disks was around $3.5 \mathrm{~mm}$ so that 
(a)
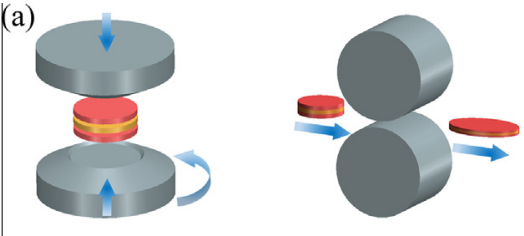

HPT

$3.5 \mathrm{~mm} \rightarrow 1.2 \mathrm{~mm}$

(b)

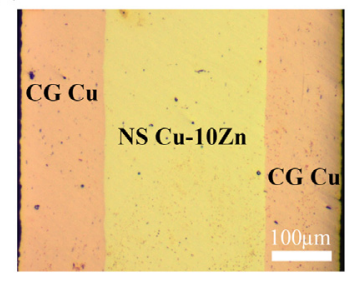

$1.2 \mathrm{~mm} \rightarrow 0.6 \mathrm{~mm}$

(c)
Rolling $\longrightarrow$ Annealing

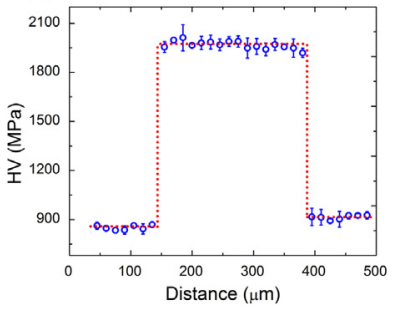

Figure 1. (a) Schematic illustration of fabrication of CG/NS/CG sandwich materials. Deformation history of thickness reduction is also provided below the corresponding step. (b) Optical microscopy observation of as-processed sandwiches with $\mathrm{NS} \mathrm{Cu}-10 \mathrm{Zn}$ volume fraction 0.47. (c) Vickers micro-hardness indentation (with loading $25 \mathrm{~g}$ ) on cross-sectional sample in (b).

sufficient thickness reduction $(\sim 83 \%)$ after processing can be achieved to form strong interfacial bonding (thickness reduction history as shown in Fig. 1a). Mechanical polishing and ultrasonic cleaning were carried out before sandwiching the disks together. High pressure torsion (HPT) was applied at room temperature with $1 \mathrm{GPa}$ for 10 revolutions at $1.5 \mathrm{rpm}$ to obtain more homogeneous deformation along the radius direction [15]. Thereafter, as-HPTed sandwiches were rolled to $0.6 \mathrm{~mm}$ from $1.2 \mathrm{~mm}$ and then annealed at $240{ }^{\circ} \mathrm{C}$ for $2 \mathrm{~h}$.

Microstructures near interfaces were characterized by FEI Quanta 3D FEG with Ion Channeling Contrast Microscopy (ICCM), JEOL-2010F Transmission Electron Microscopy (TEM) operated at $200 \mathrm{kV}$ and Electron Dispersive X-ray Spectroscopy (EDS) mapping in FEI Titan 80-300. Dog-bone shaped samples with gauge dimension of $0.6 \times 2 \times 8.4 \mathrm{~mm}^{3}$ were cut from the middle of sandwiches and tested under uniaxial tension at a strain rate of $9 \times 10^{-4} \mathrm{~s}^{-1}$. Scanning electron microscopy (SEM) was used to examine fracture surface and interface.

Three groups of samples with varying volume fraction of central NS Cu-10Zn layer (A: 0.10, B: 0.22, C: 0.47) were fabricated. Figure $1 \mathrm{~b}$ shows a typical optical micrograph (cross-sectional view) of sample C. Different colour contrast clearly indicates three layers with two sharp interfaces. As shown in Figure 1c, microhardness within each layer is rather homogeneous, while there are abrupt transitions at the interfaces, indicating a great difference of yield strength between central and outer layers. These hardness levels in each layer do not change much with variation of volume fractions.

Figure $2 \mathrm{a}$ is a channeling contrast image showing the typical microstructures near the interface. On the left side is typical CG Cu with a grain size of $\sim 4 \mu \mathrm{m}$, while on the right side is NS bronze with a grain (sub-grain) size of $\sim 100 \mathrm{~nm}$. Magnified image of the NS/CG interface is shown in Figure $2 b$, which reveals a void-free transition from NS bronze to $\mathrm{CG} \mathrm{Cu}$. The exact $\mathrm{Cu} /$ bronze interface is hard to tell in TEM and can be identified by EDS mapping. Figure 2c is a typical high-angle annular darkfield (HAADF) image including a $\mathrm{Cu} /$ bronze interface, whose precise location is unknown. EDS mappings in Figure $2 \mathrm{~d}$ and e show the elemental distribution of $\mathrm{Cu}$ and $\mathrm{Zn}$ and resolve the exact interface (marked by dotted lines) in Figure 2c. Concentrations of $\mathrm{Zn}$ are measured as $0.64 \mathrm{wt} . \%$ in the left side and $10.22 \mathrm{wt} . \%$ in the right side, respectively. This agreement of specific composition in $\mathrm{Cu}$ and bronze implies no significant bulk diffusion from each side during sample processing. Therefore, Figure $2 \mathrm{c}-\mathrm{e}$ confirm the generation of well-bonded and sharp $\mathrm{Cu} /$ bronze interface.

Figure 3a shows the tensile stress-strain curves of laminated samples, pure $\mathrm{CG} \mathrm{Cu}$ and NS bronze samples. Pure samples were made from the sandwiches by polishing off other layers. The yield strengths $(0.2 \%$-strain offset stress $)$ for samples A, B and $\mathrm{C}$ were measured as $142 \mathrm{MPa}$, $201 \mathrm{MPa}$ and $266 \mathrm{MPa}$, respectively, while their uniform elongation (engineering strain) were $27.1 \%, 19.6 \%$ and $12.3 \%$, respectively. The yield strength of the laminate sample increases and its tensile ductility decreases with increasing volume fraction of central NS bronze layer. Note that the uniform plastic deformation of NS bronze layer in sandwich is much higher $(>12 \%)$ than that $(\sim 0.7 \%)$ of its pure counterpart. This is because its early necking tendency was constrained by the stable $\mathrm{CG} \mathrm{Cu}$ layers from both sides via the two interfaces.

In the rule of mixture (ROM) for laminated composite structure, yield strength $\left(\sigma_{y s}\right)$, strain hardening $(d \sigma / d \varepsilon)$ and uniform elongation $\left(\varepsilon_{U E}\right)$ (true strain) are expressed as $[2,16,17]$

$\sigma_{y s}=\sum V_{i} \sigma_{i, y s}^{\prime}$

$\frac{d \sigma}{d \varepsilon}=\sum V_{i} \frac{d \sigma_{i}}{d \varepsilon}$

$\varepsilon_{U E}=\frac{\sum V_{i} \sigma_{i, U E} \varepsilon_{i, U E}}{\sum V_{i} \sigma_{i, U E}}$

where $V_{i}$ is the volume fraction of component $i, \sigma_{i, y s}^{\prime}$ is the flow stress of component $i$ alone at $0.2 \%$ plastic strain of the composite sample [2], $\sigma_{i}$ is the true stress of component $i, \sigma$ and $\varepsilon$ represent the true stress and true strain of integrated composite sample, $\sigma_{i, U E}, \varepsilon_{i, U E}$ stand for the true stress and true strain of component $i$ at necking point.

It is found that the yield strength of laminate samples agrees well with the ROM (Eq. (1)), as shown in Figure $3 b$. Comparisons of strain hardening and uniform elongation (true strain) between experimental results and theoretical prediction from ROM are shown in Figure $3 \mathrm{c}$ and $\mathrm{d}$. When calculating the strain-hardening rate, we assume a constant engineering stress after necking for pure bronze. This assumption provides the maximum applied stress that a standalone bronze layer can sustain according to Considère criterion. Namely, the derived strain-hardening rate is the upper limit based on ROM and would give a conservative comparison to experimental observation. For Eq. (3), it is also assumed that during the uniform elongation, the strain hardening of each component can be expressed by Hollomon law of $\sigma_{i}=k_{i} \varepsilon^{n_{i}}$ [16]. In fact, the Hollomon law has been used on NS materials in compression test $[18,19]$ despite limit reports of applications for tensile tests due to the plasticity instability. Figure $3 \mathrm{c}$ shows that the strain-hardening rate of the 

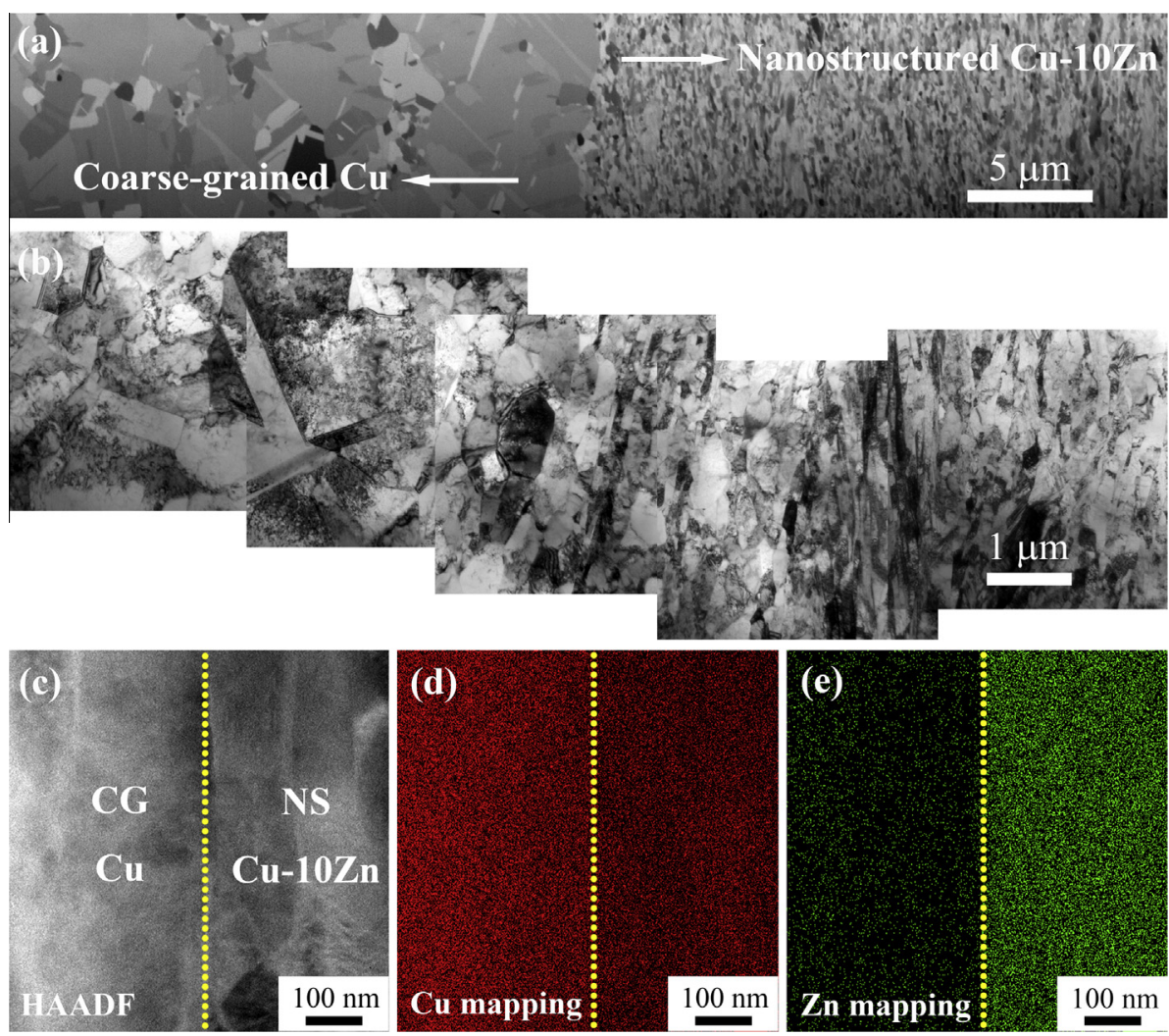

Figure 2. (a) ICCM of a typical NS/CG interface in sandwich ( $7^{\circ}$ tilt of sample while imaging). (b) TEM observation of as-processed interface. (c) HAADF imaging of an enlarged area around $\mathrm{Cu} / \mathrm{Cu}-10 \mathrm{Zn}$ interface by STEM. EDS mapping of (d) $\mathrm{Cu}$ and (e) $\mathrm{Zn}$ in corresponding regions in (c).
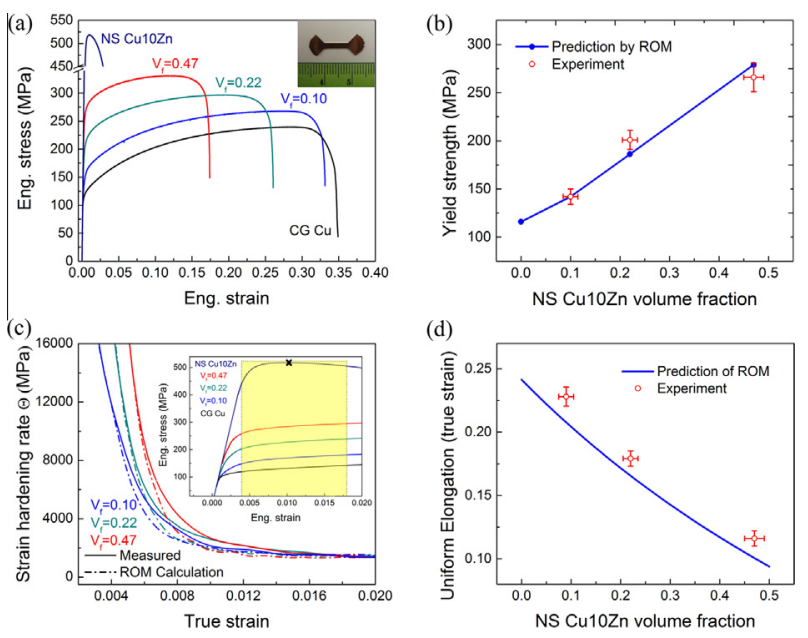

Figure 3. (a) Uniaxial tensile engineering strain-stress curves for pure $\mathrm{CG} \mathrm{Cu}, \mathrm{NS} \mathrm{Cu}-10 \mathrm{Zn}$ and sandwiches with various compositions. Inset shows the dimension of test samples. (b) Yield strength versus volume fraction of $\mathrm{NS} \mathrm{Cu}-10 \mathrm{Zn}$ from tensile tests and prediction from ROM. (c) Strain hardening curves from tensile tests and calculation based on ROM. Inset is the magnified tensile curve at low strains where extra strain hardening (yellow shade) occurs compared to ROM. Black crossover stands for the necking strain in pure $\mathrm{Cu}-10 \mathrm{Zn}$. (d) Uniform elongation versus volume fraction of $\mathrm{NS} \mathrm{Cu}-10 \mathrm{Zn}$ from tensile tests and prediction from ROM. (For interpretation of the references to color in this figure legend, the reader is referred to the web version of this article.)

laminate structure (solid) is higher than that predicted by ROM (dash-dot) at strains around necking of pure NS bronze layer. This occurs only at a limited intermediate

strain range as marked by yellow shade in the inset of Figure $3 c$. The underlying mechanism of this extra strain hardening is discussed later. Consequently, the uniform elongations of laminate samples are measured higher than what is predicted by ROM (Fig. 3d).

The integrity of interface after tensile testing was examined by SEM. Figure $4 \mathrm{a}$ is an overview of a sample, showing no inner cracks or failure through the entire uniformly elongated region except the fracture at the end. Fracture debonding should be a post-necking process due to high strain localization. In this work, the specific requirement for interface strength is only to maintain its integrity during uniform elongation until the strain where mismatch between layers and its effect on mechanical properties can

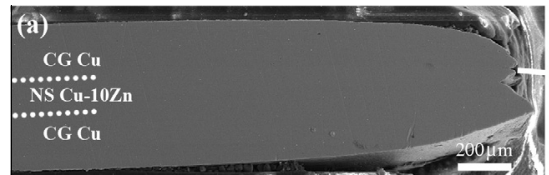

(c)

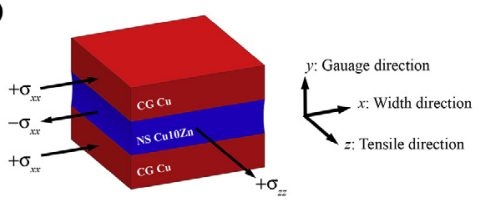

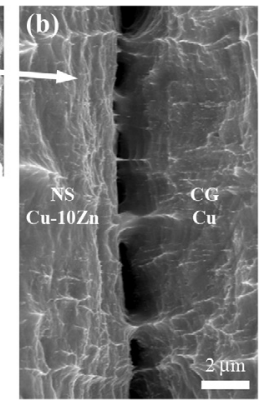

Figure 4. (a) Cross-sectional overview of the sandwich piece after test. (b) Typical morphology at lateral fracture interface in (a). (c) Schematic illustration of stress status of laminates around the strain level where the middle NS layer tends to shrink while outer CG layers stabilize it. 
be activated $(<2 \%$, as shown Fig. $3 c)$. In addition, the fracture surface shown in Figure $4 \mathrm{~b}$ reveals a frequently-seen dimple-like feature in NS/CG interface vertical to tensile direction, suggesting the strong interactions between NS/ CG layers even after necking. All of above characteristics imply well-bonded interfaces between NS and CG layers.

Pure NS bronze has a limited tensile ductility of only $0.7 \%$ due to the lack of sufficient strain hardening. However, when sandwiched by $\mathrm{CG} \mathrm{Cu}$, its uniform elongation was increased up to over 27\% (Fig. 3a). Recent modeling work of structures with grain-size difference [20] also revealed enhanced ductility in an otherwise low-ductility layer. $\mathrm{CG} \mathrm{Cu}$ has considerable strain hardening capability and therefore constrains the NS layer to postpone its necking by preventing the early-emerging tensile instability. Under such constraint, the NS bronze layer should be able to uniformly deformed further [5,21]. In addition, the mutual constraint of NS bronze layer and $\mathrm{CG} \mathrm{Cu}$ layer actually adds extra strain hardening, as shown in Figure 3c. The proposed reason is as follows. A standalone NS bronze layer will start early necking at very low strain by fast local lateral shrinkage. When sandwiched by $\mathrm{CG} \mathrm{Cu}$, this necking process is quickly suppressed by the stable outer layers at both sides. Therefore, we refer to this as "virtual necking" since it could not proceed very far. The instable middle layer and stable outer layers mutually constrain each other as the interface still holds them together, which converts uniaxial applied tensile stress to bi-axial stresses (Fig. 4c). As a result, more dislocations will be accumulated around the interface in order to accommodate the mechanical incompatibility across the interface, which produces the observed extra strain hardening. It follows that the strain-hardening rate of our laminate structure under this intermediate strain level can be described by modifying Eq. (2) as

$\frac{d \sigma}{d \varepsilon}=\sum V_{i} \frac{d \sigma_{i}}{d \varepsilon}+\Delta \Theta$

where $\Delta \Theta$ is the extra strain hardening in addition to what is predicted by conventional ROM. This observation of extra hardening is consistent with the recent discoveries in GS IF-steel [5]. In addition, extended elastic-plastic transition due to the large yield stress mismatch, as another form of mechanical incompatibility, may also contribute more or less to this phenomenon. But careful examination of magnified tensile curve (Fig. 3c inset) reveals those strain levels (yellow shade) for extra hardening are far away from the supposed elastic-plastic transition region $(\sim 0.1 \%-0.4 \%$ in Fig. $3 c$ inset) with comparison to virtual necking strain (black crossover). Therefore, the extra hardening in this study is majorly ascribed to the mismatched tensile instabilities across interfaces.

A big difference in the mechanical behaviors of the laminate structured materials and the GS IF steel reported earlier [5] is that no strain hardening up-turn is observed here. The reason for this difference is not clear and needs further study. One possible reason could be the differences of interface nature in two cases. As reported earlier and this work, the dislocation accumulation mainly occurs near the interfaces. Interfaces in laminates are stationary due to sharp discrepancy across layers and homogenous microstructure within each layer. Dislocation accumulation occurs only near the fixed interface and ceases when saturation of dislocation density is locally reached. Therefore, it cannot produce enough strain hardening for the whole sample. In contrast, interfaces in the GS IF steel is dynamically migrated and then allows the deposition of highdensity of dislocations over their entire migrating path [5]. This makes the strain hardening free from the restriction of localized dislocation saturation and able to achieve more, which results in an up-turn characteristic.

In summary, HPT followed by rolling and annealing was used to produce laminate materials by sandwiching a NS layer between CG layers. Uniaxial tensile test is performed and reveals an extra strain hardening that leads to larger uniform elongation than what is predicted by conventional ROM. The interface is well bonded to maintain the overall uniform deformation for the whole sample. This preliminary work indicates a critical role played by interfaces in the mechanical behaviors of laminate and gradient structured materials.

We are grateful for financial support of 973 programs (grants 2012CB932203, 2012CB937500, and 6138504), National Natural Science Foundation of China (grants 11172187, 11002151, 11072243, 51301187 and 50571110), the Pangu Foundation, the Program for New Century Excellent Talents in University (NCET-12-0372) and the US Army Research Office (grants W911NF-09-1-0427 and W911QX-08-C-0083). We also acknowledge Analytical Instrumentation Facility (AIF) at North Carolina State University, which is supported by the State of North Carolina and the National Science Foundation.

[1] T.H. Fang, W.L. Li, N.R. Tao, K. Lu, Science 331 (2011) $1587-1590$.

[2] X.L. Wu, P. Jiang, L. Chen, J.F. Zhang, F.P. Yuan, Y.T. Zhu, Mater. Res. Lett. (2014) 1-7.

[3] X.L. Lu, Q.H. Lu, Y. Li, L. Lu, Sci. Rep. 3 (2013).

[4] A. Jérusalem, W. Dickson, M.J. Pérez-Martín, M. Dao, J. Lu, F. Gálvez, Scripta Mater. 69 (2013) 773-776.

[5] X. Wu, P. Jiang, L. Chen, F. Yuan, Y.T. Zhu, Proc. Natl. Acad. Sci. U.S.A. 111 (2014) 7197-7201.

[6] K. Lu, Science 345 (2014) 1455-1456.

[7] D.K. Yang, P. Cizek, D. Fabijanic, J.T. Wang, P.D. Hodgson, Acta Mater. 61 (2013) 2840-2852.

[8] Y. Wei, Y. Li, L. Zhu, Y. Liu, X. Lei, G. Wang, Y. Wu, Z. Mi, J. Liu, H. Wang, H. Gao, Nat. Commun. 5 (2014).

[9] A.Y. Chen, D.F. Li, J.B. Zhang, H.W. Song, J. Lu, Scripta Mater. 59 (2008) 579-582.

[10] X.C. Liu, H.W. Zhang, K. Lu, Science 342 (2013) 337-340.

[11] S. Suresh, Science 292 (2001) 2447-2451.

[12] I.J. Beyerlein, J.R. Mayeur, S. Zheng, N.A. Mara, J. Wang, A. Misra, Proc. Natl. Acad. Sci. U.S.A. 111 (2014) 43864390.

[13] Y.H. Zhao, Y.T. Zhu, X.Z. Liao, Z. Horita, T.G. Langdon, Appl. Phys. Lett. 89 (2006) 121906.

[14] K. Barmak, A. Gungor, C. Cabral, J.M.E. Harper, J. Appl. Phys. 94 (2003) 1605-1616.

[15] X.H. An, S.D. Wu, Z.F. Zhang, R.B. Figueiredo, N. Gao, T.G. Langdon, Scripta Mater. 63 (2010) 560-563.

[16] S.L. Semiatin, H.R. Piehler, Metall. Trans. A 10 (1979) 85 96.

[17] D. Nyung Lee, Y. Keun Kim, J. Mater. Sci. 23 (1988) 1436 1442.

[18] D. Jia, Y.M. Wang, K.T. Ramesh, E. Ma, Y.T. Zhu, R.Z. Valiev, Appl. Phys. Lett. 79 (2001) 611-613.

[19] Y.M. Wang, J.Y. Huang, T. Jiao, Y.T. Zhu, A.V. Hamza, J. Mater. Sci. 42 (2007) 1751-1756.

[20] L. Jianjun, A.K. Soh, Model. Simul. Mater. Sci. Eng. 20 (2012) 085002.

[21] X.L. Wu, Y.T. Zhu, Y.G. Wei, Q. Wei, Phys. Rev. Lett. 103 (2009) 205504. 